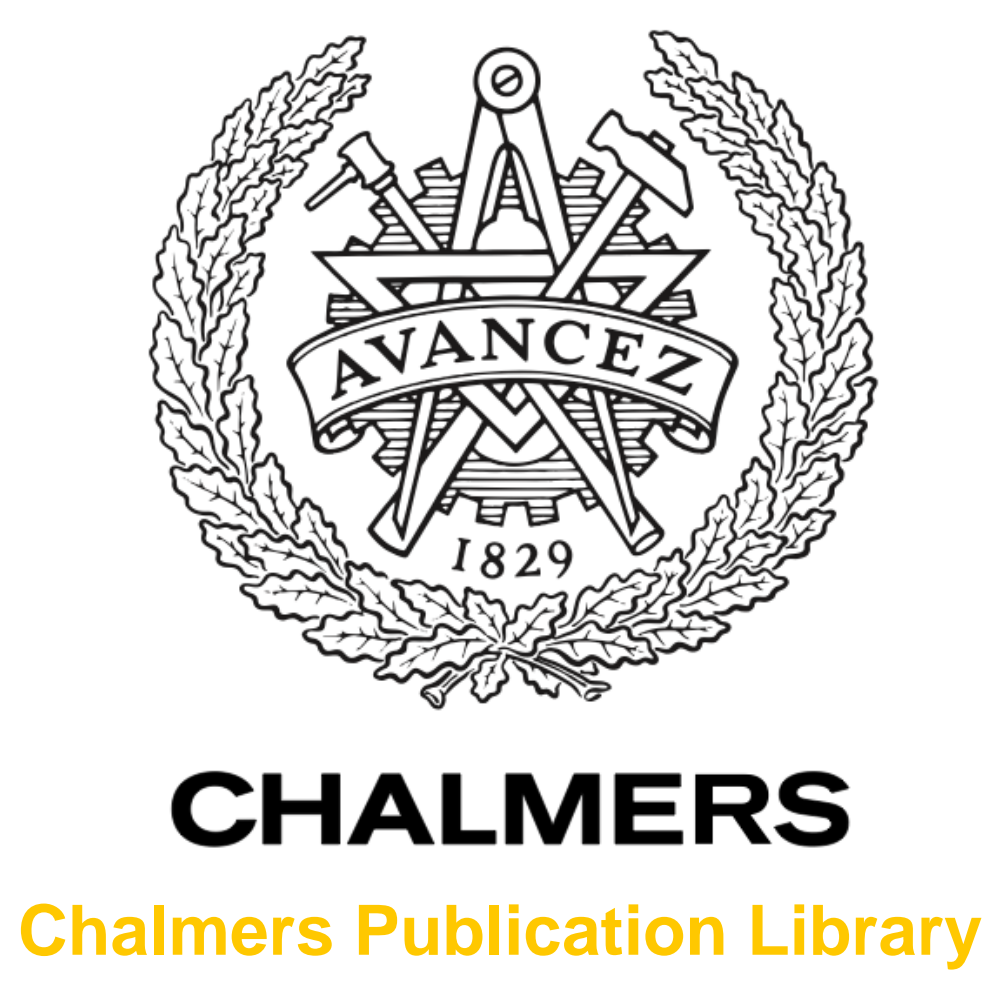

Three Super-Earths Transiting the Nearby Star GJ 9827

This document has been downloaded from Chalmers Publication Library (CPL). It is the author's version of a work that was accepted for publication in:

Astronomical Journal (ISSN: 0004-6256)

Citation for the published paper:

Niraula, P. ; Redfield, S. ; Dai, F. et al. (2017) "Three Super-Earths Transiting the Nearby

Star GJ 9827". Astronomical Journal, vol. 154(6), pp. 266.

http://dx.doi.org/10.3847/1538-3881/aa957c

Downloaded from: http://publications.lib.chalmers.se/publication/253459

Notice: Changes introduced as a result of publishing processes such as copy-editing and formatting may not be reflected in this document. For a definitive version of this work, please refer to the published source. Please note that access to the published version might require a subscription.

Chalmers Publication Library (CPL) offers the possibility of retrieving research publications produced at Chalmers University of Technology. It covers all types of publications: articles, dissertations, licentiate theses, masters theses, conference papers, reports etc. Since 2006 it is the official tool for Chalmers official publication statistics. To ensure that Chalmers research results are disseminated as widely as possible, an Open Access Policy has been adopted.

The CPL service is administrated and maintained by Chalmers Library. 


\title{
Three Super-Earths Transiting the Nearby Star GJ 9827
}

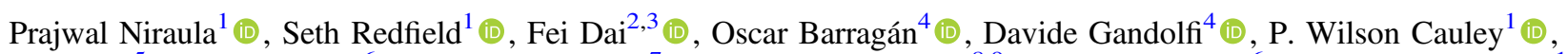

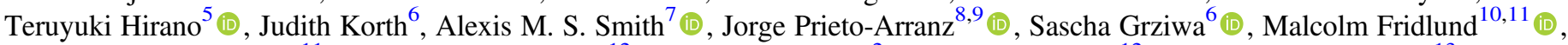
Carina M. Persson ${ }^{11}$ (i), Anders Bo Justesen ${ }^{12}$ (i), Joshua N. Winn ${ }^{2}$ (i), Simon Albrecht ${ }^{12}$ (i), William D. Cochran ${ }^{13}$ (1), Szilard Csizmadia ${ }^{7}$, Girish M. Duvvuri ${ }^{1}\left(\mathbb{0}\right.$, Michael Endl $^{13}$ (1), Artie P. Hatzes ${ }^{14}$, John H. Livingston ${ }^{15}$, Norio Narita ${ }^{15,16,17}$ (1), David Nespral $^{8,9}$, Grzegorz Nowak ${ }^{8,9}$ (D), Martin Pätzold ${ }^{6}$, Enric Palle ${ }^{8,9}$ (1) , and Vincent Van Eylen ${ }^{10}$

${ }^{1}$ Astronomy Department and Van Vleck Observatory, Wesleyan University, Middletown, CT 06459, USA; pniraula@wesleyan.edu ${ }^{2}$ Department of Astrophysical Sciences, Princeton University, 4 Ivy Lane, Princeton, NJ 08544, USA

3 Department of Physics and Kavli Institute for Astrophysics and Space Research, Massachusetts Institute of Technology, Cambridge, MA 02139, USA 4 Dipartimento di Fisica, Universitá di Torino, via P. Giuria 1, I-10125 Torino, Italy

${ }^{5}$ Department of Earth and Planetary Sciences, Tokyo Institute of Technology, 2-12-1 Ookayama, Meguro-ku, Tokyo 152-8551, Japan

${ }^{6}$ Rheinisches Institut für Umweltforschung an der Universität zu Köln, Aachener Strasse 209, D-50931 Köln, Germany

${ }^{7}$ Institute of Planetary Research, German Aerospace Center, Rutherfordstrasse 2, D-12489 Berlin, Germany

${ }^{8}$ Instituto de Astrofísica de Canarias, C/ Vía Láctea s/n, E-38205 La Laguna, Spain

${ }^{9}$ Departamento de Astrofísica, Universidad de La Laguna, E-38206 La Laguna, Spain

${ }^{10}$ Leiden Observatory, University of Leiden, P.O. Box 9513, 2300 RA, Leiden, The Netherlands

${ }^{11}$ Department of Space, Earth and Environment, Chalmers University of Technology, Onsala Space Observatory, SE-439 92 Onsala, Sweden

${ }^{12}$ Stellar Astrophysics Centre (SAC), Department of Physics and Astronomy, Aarhus University, Ny Munkegade 120, DK-8000, Denmark

${ }^{13}$ Department of Astronomy and McDonald Observatory, University of Texas at Austin, 2515 Speedway, Stop C1400, Austin, TX 78712, USA

${ }^{14}$ Thüringer Landessternwarte Tautenburg, Sternwarte 5, D-07778 Tautenberg, Germany

15 Department of Astronomy, University of Tokyo, 7-3-1 Hongo, Bunkyo-ku, Tokyo 113-0033, Japan

${ }^{16}$ Astrobiology Center, NINS, 2-21-1 Osawa, Mitaka, Tokyo 181-8588, Japan

${ }^{17}$ National Astronomical Observatory of Japan, NINS, 2-21-1 Osawa, Mitaka, Tokyo 181-8588, Japan

Received 2017 September 1; revised 2017 October 20; accepted 2017 October 20; published 2017 December 1

\begin{abstract}
We report on the discovery of three transiting planets around GJ 9827. The planets have radii of $1.75 \pm 0.18$, $1.36 \pm 0.14$, and $2.11_{-0.21}^{+0.22} R_{\oplus}$, and periods of $1.20896,3.6480$, and 6.2014 days, respectively. The detection was made in Campaign 12 observations as part of our $K 2$ survey of nearby stars. GJ 9827 is a $V=10.39$ mag K6V star at a distance of $30.3 \pm 1.6$ parsecs and the nearest star to be found hosting planets by Kepler and K2. The radial velocity follow-up, high-resolution imaging, and detection of multiple transiting objects near commensurability drastically reduce the false positive probability. The orbital periods of GJ $9827 \mathrm{~b}$, c, and d planets are very close to the 1:3:5 mean motion resonance. Our preliminary analysis shows that GJ 9827 planets are excellent candidates for atmospheric observations. Besides, the planetary radii span both sides of the rocky and gaseous divide, hence the system will be an asset in expanding our understanding of the threshold.
\end{abstract}

Key words: planets and satellites: detection - stars: individual (GJ 9827, K2-135)

Supporting material: data behind figure

\section{Introduction}

Temporal monitoring of neighboring stars (e.g., within 100 parsecs and therefore relatively bright) provides an opportunity to search for nearby planetary systems that are optimal for follow-up studies. This includes favorable conditions to characterize the system as a whole, particularly properties that can be directly linked to the planetary atmosphere and habitability, such as the stellar UV emission (Linsky et al. 2014), stellar wind strength (Wood et al. 2005), and stellar magnetic field structure (Alvarado-Gómez et al. 2016). As the Kepler mission and ground-based radial velocity (RV) searches have shown, terrestrial planets are ubiquitous (Howard et al. 2012; Fressin et al. 2013). The sample of terrestrial exoplanets will continue to grow with dedicated ground and space-based surveys (e.g., $K 2$, and in the future with the Transiting Exoplanet Survey Satellite; Ricker et al. 2015). A major scientific endeavor related to this population of planets will be the evaluation of habitability and a search for biosignatures. It is precisely in these bright, nearby systems where the atmospheric measurements will be the most sensitive, and the question of habitability will be examined in the greatest detail in the decades to come.

$K 2$, the repurposed Kepler mission, has continued the legacy of planet discovery by its predecessor (Howell et al. 2014). While the $K 2$ fields can only be monitored for about 80 days, and thereby limiting discoveries to relatively short period transiting objects, its ability to observe different parts of the ecliptic plane and choice of more diverse targets has led to some intriguing discoveries. Many planetary candidates have been reported (e.g., Crossfield et al. 2016) along with the first detection of transiting bodies orbiting the white dwarf WD $1145+017$ (Vanderburg et al. 2015). K2 also continues to find multiplanetary systems, which are of interest for the study of planetary architecture and formation. Sinukoff et al. (2016) reported the detection of 11 multiplanetary systems from $K 2$ Campaigns 1 and 2. However, there are few such systems around nearby stars (Armstrong et al. 2015; Crossfield et al. 2015; Gandolfi et al. 2017), and only a handful around brighter stars that are suitable for spectroscopic characterization.

We have detected a new planetary system hosted by a K6V star, GJ 9827 (EPIC 246389858 (K2-135)). At $30.3 \pm 1.6$ 
parsecs, it is the nearest planetary system detected by Kepler or $K 2$. Our analysis of the Kepler light curve demonstrates the presence of three super-Earths of radii around GJ 9827 (K2-135b, $\mathrm{K} 2-135 \mathrm{c}$, and K2-135d). We will use the designation of superEarth for planets with radii from 1.25 to $2 R_{\oplus}$ (e.g., Batalha et al. 2013), although note that the precise limits of this range are largely arbitrary and GJ $9827 \mathrm{~d}$ lies just above the upper bound of this designation. The planets orbit at a distance of $0.020 \pm 0.002,0.041 \pm 0.003$ and $0.059_{-0.005}^{+0.004}$ au corresponding to orbital periods of $1.208957_{-0.000013}^{+0.000012}, 3.64802 \pm 0.00011$, and $6.20141_{-0.00010}^{+0.00012}$ days respectively. The planetary system is tightly packed, and the periods are close to 1:3:5 commensurability. In addition to the fact that GJ 9827 is a relatively bright star, the planets occur on both sides of the rocky and gaseous threshold of $\sim 1.5 R_{\oplus}$ (Weiss \& Marcy 2014; Rogers 2015). Hence the system is likely to be a great asset in understanding the nature of this threshold, and could potentially exhibit a range of densities like the Kepler-36 planets (Carter et al. 2012).

GJ 9827 planets are great candidates for atmospheric studies. In the past, ground-based telescopes, along with the Hubble Space Telescope and Spitzer, have been successfully used to characterize the atmospheres of hot Jupiters (Charbonneau et al. 2002; Knutson et al. 2008; Redfield et al. 2008; Sing et al. 2015). With the James Webb Space Telescope (JWST), this territory will be extended into the super-Earth regime (Deming et al. 2009). Bright, nearby planetary systems, like GJ 9827, will provide excellent opportunities to probe the conditions of super-Earth atmosphere.

\section{Observations and Data Analysis}

GJ 9827 (EPIC 246389858) was proposed by our team (PI Redfield) as part of a Campaign 12 survey of nearby stars (GO-12039), and in three other programs: GO-12071, PI Charbonneau; GO-12049 PI Quintana; and GO-12123 PI Stello. The star was observed for a total of 78.89 days from 2016 December 15 to 2017 March 4 at the boundary of constellation Aquarius and Pisces at R.A. of 23:27:04.835 and decl. $-01: 17: 10.58$ in long cadence mode.

\subsection{K2 Observations}

We implement a data reduction pipeline to detrend the systematic $K 2$ noise. We follow the protocol to decorrelate the data against its arclength (1D) using one of the three standard stars from the Campaign (e.g., Vanderburg \& Johnson 2014; Vanderburg et al. 2016). These standard pointing stars are chosen such that their centroid can be found with better precision than an average star in the field. Among these three standards, the light curve is decorrelated with the star whose centroid variation over time is best fit with a fifth-degree polynomial, in this case, EPIC 246292491. Besides, we use a modified version of Van Eylen et al.'s (2016) publicly available code, ${ }^{18}$ which detrends the light curve by a simultaneous second-order fit for both the centroid coordinates and time, also allowing for a cross-term between two centroids. The k2photometry pipeline yields a flattened light curve. In our implementation, the final transit removed light curve from k2photometry has a standard deviation of $77 \mathrm{ppm}$ compared to $106 \mathrm{ppm}$ from Vanderburg's method. Thus in Figure 1, we show the detrended flux obtained from

\footnotetext{
18 https://github.com/vincentvaneylen/k2photometry
}

Vanderburg's method and the normalized light curve from $\mathrm{k} 2$ photometry. These values are higher by a factor of $\sim 2$ than the expected calculated rms values of 39.2 for $10.5 \mathrm{~V}$ magnitude star, ${ }^{19}$ which is expected due to pointing induced errors for $K 2$.

As for some of the unique aspects of our pipeline, we take the median value in each frame as the background. In order to avoid the effect of the outliers, we perform an iterative spline fitting, rejecting $3 \sigma$ outliers until convergence. Finally, the background is subtracted from the photometric flux. We reject the data with bad quality flags, which resulted in excluding around $15 \%$ of the data flagged for thruster firing, Agrabrightening, cosmic-ray detection, and pipeline outlier detection. This has led to two instances where the transits are completely missing (refer to Figure 1). We did a follow-up test with different aperture sizes from which a circular aperture of $\sim 20^{\prime \prime}$ radius is chosen. Initially, we define our aperture as the largest contiguous region above twice the median. From this we calculate the centroid of the star. However, the calculated centroid of the star does not coincide with the FITS coordinates probably because GJ 9827 is a high proper motion star (Stephenson 1986).

Clear stellar modulation, presumably associated with stellar rotation, is evident in the detrended light curve of Figure 1. After we remove the first five days of data, which show anomalies probably related to thermal settling, the autocorrelation function (McQuillan et al. 2013) of the detrended light curve exhibits a peak at $16.9_{-1.51}^{+2.14}$ days, which is consistent with our reported $v \sin i$ value of $2 \pm 1 \mathrm{~km} \mathrm{~s}^{-1}$ assuming a stellar inclination of $90^{\circ}$. However, we also observed an almost comparable secondary peak at 29 days, which is congruous with the value of $1.3_{-1.3}^{+1.5} \mathrm{~km} \mathrm{~s}^{-1}$ reported in Houdebine et al. (2016). A longer baseline of observations would help to determine the true stellar rotation period.

We perform a Box Least-Squared (BLS; Kovács et al. 2002) search on the flattened light curve to detect the presence of any planetary signals. Once a transit signal is identified, it is fitted and removed from the light curve. In this fashion, we iteratively run the BLS algorithm on the light curve for further detection of additional transit signals. In GJ 9827, this showed a presence of three transiting planets. A simultaneous fit for all of the three identified transits is then performed with the batman model supersampled by a factor of 15, and adjusted for K2's long cadence (Kreidberg 2015). We use the affine invariant MCMC method implemented in emcee (ForemanMackey et al. 2013) with 100 walkers for 30,000 steps; of this, the first 22,500 steps were removed as burn-in. The rest of the data is used to build the posterior distributions and estimate the uncertainties in our transit parameters. The fits are shown in Figure 2.

We use uniform priors for the period, time of conjunction, scaled planet radius, and impact parameter for all three planets. For limb darkening parameters, we use triangular sampling suggested by Kipping (2013). We additionally use Sing (2010) to introduce Gaussian priors on limb darkening based on the stellar parameters. We use a mean value of 0.5782 for $u_{1}$, and 0.1428 for $u_{2}$, both with 0.1 standard deviation. Since this is a short period multiplanetary system, we assume tidal circularization of the orbits and adopt a fixed eccentricity of $e=0$ for all three planets (Van Eylen \& Albrecht 2015). As for the

\footnotetext{
${ }^{19}$ https://keplergo.arc.nasa.gov/CalibrationSN.shtml
} 

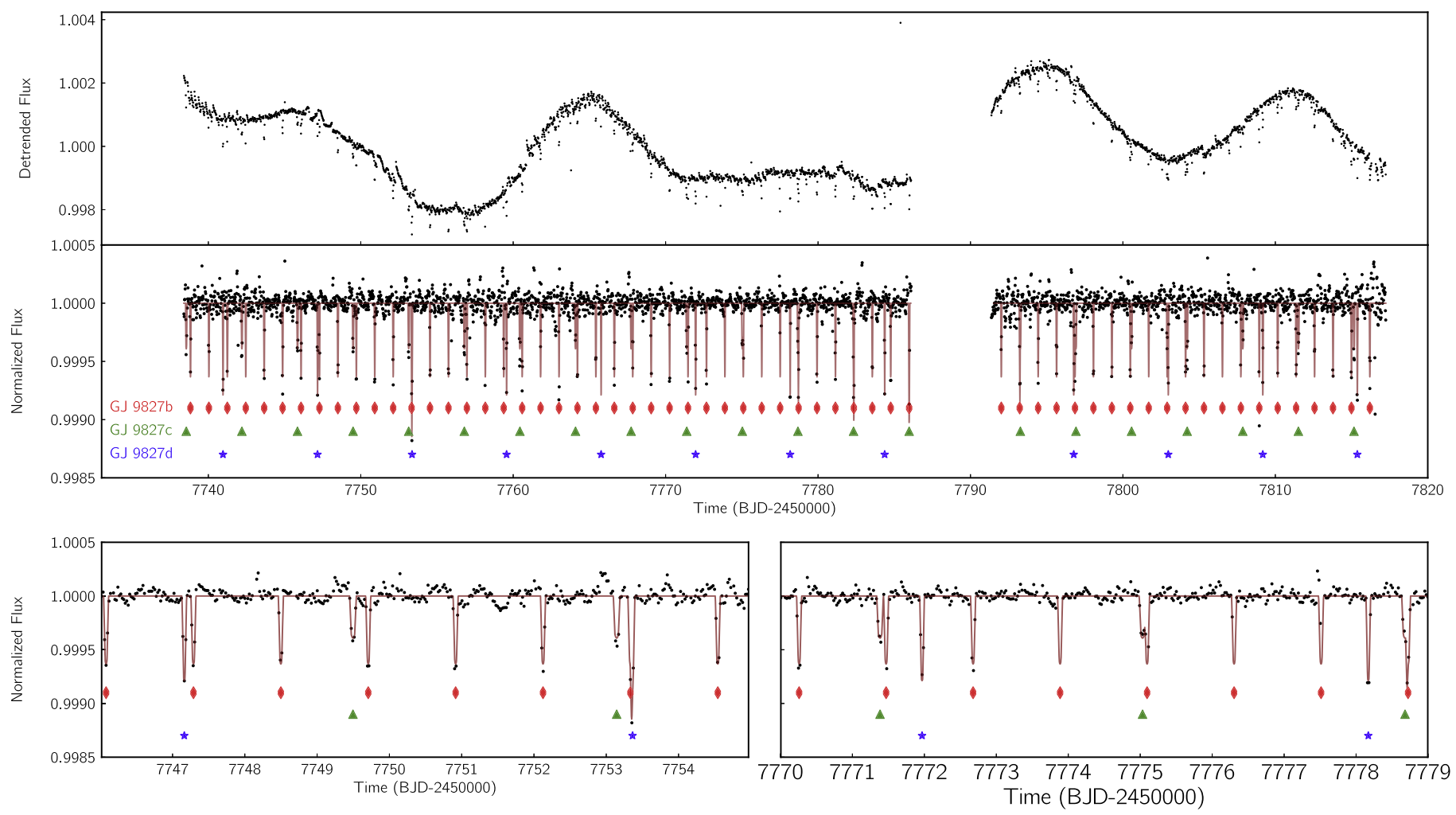

Figure 1. Detrended and normalized $K 2$ light curve of EPIC 246389858. Transits of each planet are marked, and the combined fit (brown line) at a finer sampling rate for all transits based on MCMC fits, presented in Table 1, is shown. The bottom left and bottom right figures zoom into two different sections of the data. The data used to create this figure are available.

Table 1

Planetary Parameters of GJ 9827 b, c, and d

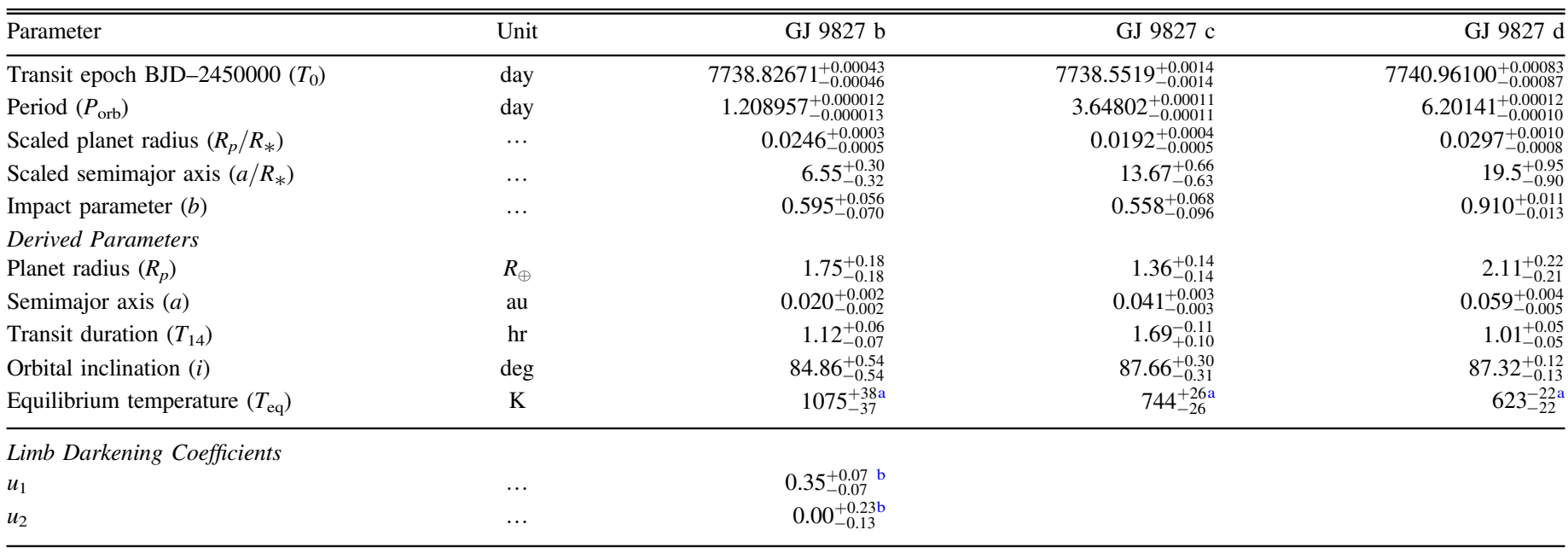

Notes. The values of eccentricity for all three planets is fixed at zero.

${ }^{\text {a }}$ We calculate equilibrium temperature as $T_{\mathrm{eq}}=T_{*} \sqrt{R_{*} / 2 a}(1-\alpha)^{1 / 4}$, where Bond Albedo $(\alpha)$ is adopted at 0.3 .

${ }^{\mathrm{b}}$ A single set of limb darkening parameters is fitted for three different transit light curves.

scaled semimajor axis of GJ $9827 \mathrm{c}$ and d, we assume they are constrained by Kepler's Third Law. As a result, we fit 15 independent variables (Table 1), and the resulting fit is shown in Figure 2. We additionally introduce a Gaussian prior based on the spectroscopically derived stellar density of $3.37 \pm 0.51 \mathrm{~g} \mathrm{~cm}^{-3}$. MCMC runs without Gaussian priors on sometimes converged to unrealistic semimajor axis values, hence the choice. From the posterior distribution, most of the variables are well constrained except for limb darkening parameters. Due to short transit duration and long integration time for $K 2$, limb darkening parameters are not expected to be well constrained (Kipping 2010). The introduction of Gaussian prior for limb darkening parameters does not noticeably affect the other fit parameters. 
Table 2

FIES RV Measurements

\begin{tabular}{lcc}
\hline \hline $\begin{array}{l}\text { BJD } \\
-2,450,000\end{array}$ & $\begin{array}{c}\mathrm{RV} \\
\left(\mathrm{km} \mathrm{s}^{-1}\right)\end{array}$ & $\begin{array}{c}\text { Error } \\
\left(\mathrm{km} \mathrm{s}^{-1}\right)\end{array}$ \\
\hline 7954.617085 & 31.7746 & 0.0033 \\
7955.612895 & 31.7724 & 0.0032 \\
7956.627456 & 31.7751 & 0.0025 \\
7964.582846 & 31.7796 & 0.0028 \\
7965.593839 & 31.7739 & 0.0032 \\
7966.573354 & 31.7728 & 0.0033 \\
7966.707233 & 31.7735 & 0.0035 \\
\hline
\end{tabular}

It is interesting to note that the transit duration is longest for GJ 9827 c, and shortest for GJ 9827 d. This is consistent with the fit's prediction that GJ $9827 \mathrm{~d}$ has a higher impact parameter than either GJ 9827 b or c. Additional independent MCMC runs were performed by our team using pyanet $i$ (Barragán et al. 2017a), with flattened light curves from independent pipelines developed in our group, and the results are within $1 \sigma$ errors. Note that the high impact parameter of GJ 9827 d suggests additional planets, if present, are likely to be nontransiting. This possibility will be explored in the follow-up RV campaign.

\subsection{Spectroscopic Observations}

We collected seven high-resolution $(R \approx 67,000)$ spectra of GJ 9827 using the FIbre-fed Échelle Spectrograph (FIES; Frandsen \& Lindberg 1999; Telting et al. 2014) mounted at the $2.56 \mathrm{~m}$ Nordic Optical Telescope (NOT) of the Roque de los Muchachos Observatory (La Palma, Spain). The follow-up was performed between 2017 July 20 and August 1 UT as part of the OPTICON observing program 2017A/064, under clear and stable weather conditions, with seeing ranging between 0 ". 5 and 0.8 . For each observation epoch, we took three consecutive subexposures of $900 \mathrm{~s}$ that were average combined using a sigma-clipping algorithm to remove cosmic-ray hits. Following the observing strategy described in Buchhave et al. (2010) and Gandolfi et al. (2013), we traced the RV drift of the instrument by acquiring $\mathrm{ThAr}$ spectra with long exposure $\left(T_{\exp }=65 \mathrm{~s}\right)$ taken immediately before and after each observation. We reduced the FIES data using standard IRAF and IDL routines, which include bias subtraction, flat fielding, order tracing and extraction, and wavelength calibration. RV measurements were extracted using the multi-order crosscorrelation technique with the RV standard star HD 190007observed with the same instrument set-up as the target objectfor which we adopted a heliocentric RV of $-30.40 \mathrm{~km} \mathrm{~s}^{-1}$, as measured by Udry et al. (1999). We report the RVs and their uncertainties in Table 2. Our measurements show no significant $\mathrm{RV}$ variation: the rms is $2.4 \mathrm{~m} \mathrm{~s}^{-1}$, which is comparable to the mean nominal uncertainty of $3.1 \mathrm{~m} \mathrm{~s}^{-1}$.

We used the coadded FIES spectrum, which has an SNR ratio of $\sim 150$ per pixel at $5500 \AA$, to derive the fundamental parameters of GJ 9827. The analysis was performed following the procedures already adopted for other $K 2$ host stars (Johnson et al. 2016; Barragán et al. 2017b; Fridlund et al. 2017; Gandolfi et al. 2017; Guenther et al. 2017). We took advantage of four different spectral analysis packages applied independently by different subgroups within our team. The four analyses provide consistent results well within the error bars. While we have no strong reason to prefer one method over the
Table 3

Stellar Parameters of GJ 9827 (EPIC 246389858 (K2-135))

\begin{tabular}{lcc}
\hline \hline Parameter & Units & Value \\
\hline$V$ mag & $\ldots$ & $10.39^{\mathrm{a}}$ \\
$J$ mag & $\ldots$ & $7.984^{\mathrm{b}}$ \\
Distance & $\mathrm{pc}$ & $30.3 \pm 1.6^{\mathrm{c}}$ \\
Spectral type & $\ldots$ & $\mathrm{K}^{\mathrm{d}}$ \\
Effective temperature $\left(T_{\mathrm{eff}}\right)$ & $\mathrm{K}$ & $4255 \pm 110^{\mathrm{d}}$ \\
Surface gravity $(\log g)$ & $\mathrm{cgs}$ & $4.70 \pm 0.15^{\mathrm{d}}$ \\
Iron abundance $([\mathrm{Fe} / \mathrm{H}])$ & $\mathrm{dex}$ & $-0.28 \pm 0.12^{\mathrm{d}}$ \\
Radius $\left(R_{*}\right)$ & $R_{\odot}$ & $0.651 \pm 0.065^{\mathrm{d}}$ \\
Mass $\left(M_{*}\right)$ & $M_{\odot}$ & $0.659 \pm 0.060^{\mathrm{d}}$ \\
$v \sin i$ & $\mathrm{~km} \mathrm{~s}^{-1}$ & $2 \pm 1^{\mathrm{d}}$ \\
Rotational period $\left(P_{\mathrm{rot}}\right)$ & day & $16.9_{-1.51}^{+2.14 \mathrm{~d}}$ \\
\hline
\end{tabular}

Notes.

a Adopted from Zacharias et al. (2013).

b Adopted from Cutri et al. (2003).

${ }^{\mathrm{c}}$ Hipparcos (van Leeuwen 2007).

d This work.

other, we adopted the results obtained using SpecMatchEmp (Yee et al. 2017). This technique relies on the use of highresolution template spectra of stars whose effective temperature $\left(T_{\text {eff }}\right)$, radius $\left(R_{\star}\right)$, and iron abundance $([\mathrm{Fe} / \mathrm{H}])$, have been accurately measured by interferometry, spectrophotometry, and spectral synthesis. We use Mann et al.'s (2015) empirical relations to derive the stellar mass. Our stellar parameters are presented in Table 3. The values are consistent with those reported by Houdebine et al. (2016).

\subsection{Limits on a Stellar Companion}

We investigate the probability that the transit signals are of a nonplanetary origin coming from a background source or a companion. Lissauer et al. (2012) estimate the false positive probability for systems with three transiting planet candidates at $<0.4 \%$ with the extremely conservative assumption of a $50 \%$ false positive rate for single planet candidates. The probabilities for detecting 1 planet +2 false positives or 2 planets +1 false positive are even lower than the 3 planet case. The short orbital periods also argue against a massive triple system, which would be dynamically unstable and produce very large transit timing variations (TTV; Lissauer et al. 2011), which are not observed (see Section 3.1).

Using high-resolution Lucky Imaging I-band observations, Jódar et al. (2013) find no evidence of a stellar companion to GJ 9827. They rule out all companions with $T_{\text {eff }} \gtrsim 3200 \mathrm{~K}$, or earlier than spectral type M4, at angular separations $\gtrsim 0$ ". 5 . The constraints are even tighter for angular separations $\gtrsim 1$ !' 0 , ruling out all companions with $T_{\text {eff }} \gtrsim 2800 \mathrm{~K}$, or earlier than spectral type $\sim$ M6.5.

We can also rule out companions with $T_{\text {eff }} \gtrsim 3200$ at any separation by assuming normal main-sequence dwarf parameters (Pecaut \& Mamajek 2013): if a bound, unresolved companion is present, anything with $T_{\text {eff }} \gtrsim 3000 \mathrm{~K}$ would result in $J \lesssim 7.95$. This is incompatible with the measured 2MASS $J$-magnitude of $J=7.984 \pm 0.020 \quad$ (Skrutskie et al. 2006). Thus, any undetected bound stellar companions to GJ 9827 must have a spectral type later than M5. Using optical and infrared photometry, including the Wide-Field Infrared Survey Explorer 3.4, 4.6, 12.0, and $22.0 \mu \mathrm{m}$ magnitudes (Wright et al. 2010), we also find no evidence of 


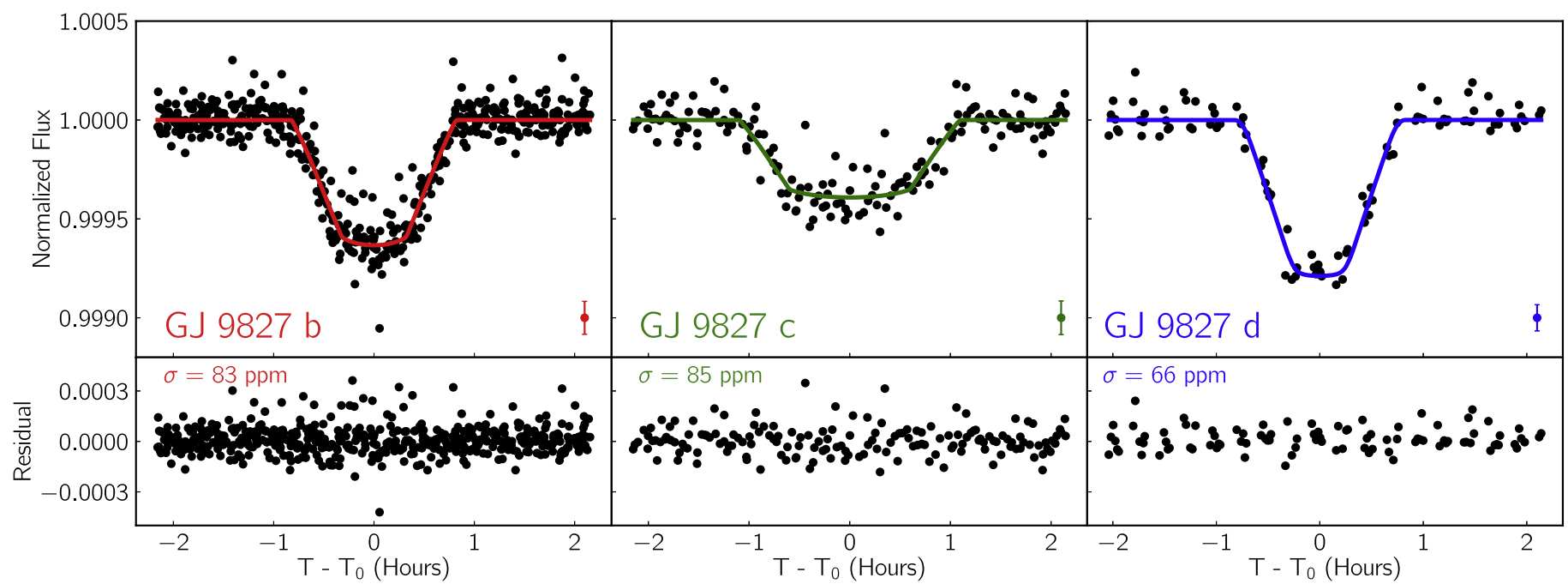

Figure 2. Model Fit of MCMC obtained parameters for GJ 9827 b (K2-135b), GJ 9827 c K2-135c, and GJ 9827 d K2-135d. The parameters are available in Table 1. Note that the normalized flux scale is kept constant for comparison. $1 \sigma$ error bars computed from the respective residuals are shown in the right-hand bottom corner for reference.
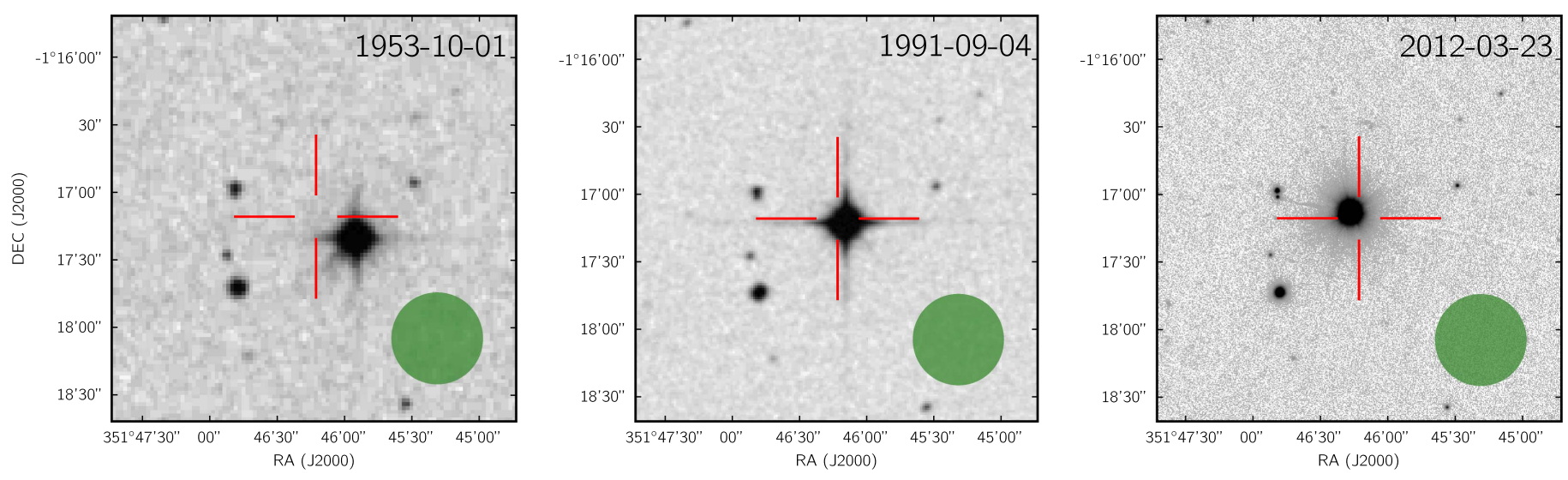

Figure 3. Archival image in $r$ band of GJ 9827 from the POSS-I and -II from years 1953 and 1991. The third image is from more recent Pan-STARRS in $g$ band from year 2012. No background objects concurrent with current position of GJ 9827 are seen in the archival image. The green circle in each image shows the $20^{\prime \prime}$ aperture size used for $K 2$ photometry; meanwhile, the reference position of GJ 9827 at the J2000 epoch is indicated with a red reticle.

any infrared excess. Although we cannot rule out the existence of a faint late-type companion, we currently favor GJ 9827 as the host star. We note that the planetary radii necessary to produce the observed transit depths are still $\sim 3-5 R_{\oplus}$ if the candidates orbit an undetected late-type companion, placing them in the mini-Neptune regime. Follow-up RV observations and high-contrast adaptive optics imaging will help confirm the nature of the planets' parent star.

Given its large proper motion $\left(\approx 400\right.$ mas $\left.\mathrm{yr}^{-1}\right)$, we are able to rule out the possibility of an unbound background contamination using the archival data. Using the STScI Digitized Sky Survey, ${ }^{20}$ we identify GJ 9827 images as early as 1953 (see Figure 3). By comparing the image to the latest epoch (2012), we determine that there is no background object coincident with its current position visible in the 1953 plate. In order to estimate the limiting magnitude of the 1953 image, we considered an object near to our target that is faint, but clearly above the detection threshold of the image. By reference to the SDSS catalog, we determined that this object has $r=19.0$ (see

$\overline{{ }^{20}}$ http://stdatu.stsci.edu/cgi-bin/dss_form
$R=10.1$ for GJ 9827). We, therefore, conclude that the 1953 plate is sensitive to objects about 9 mag fainter than GJ 9827 , and we can rule out the presence of unbound contaminants brighter than this. An equal mass eclipsing binary system with a combined magnitude of $r=19.0$ would produce at most a $125 \mathrm{ppm}$ deep signal in the light curve of GJ 9827, which is shallower than the observed transits.

\section{Discussion}

\subsection{A Closely Packed Super-Earth System}

Multi-transiting planetary systems offer more than conventional ways for characterizing the systems. Through TTV and transit duration variation (TDV), planetary masses and orbital elements in these systems can be constrained to higher precision than single transiting systems (Agol et al. 2005; Ragozzine \& Holman 2010). In addition, they provide an opportunity to test in situ versus ex situ planetary formation, which continues to be a topic of debate in the regime of superEarths (Chiang \& Laughlin 2013; Schlichting 2014; D'Angelo \& Bodenheimer 2016). 

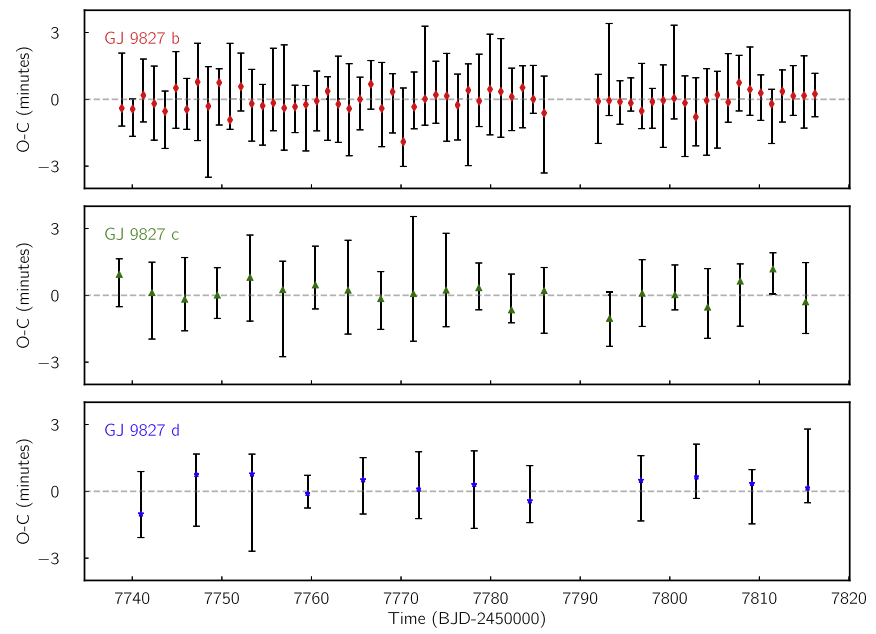

Figure 4. O-C Diagram for GJ 9827 b, c, and d. The O-C signal and errors are estimated using the MCMC fit using a model created with transit parameters. No significant TTVs greater than three minutes is detected.

No TTVs greater than 3 minutes were found for the planets GJ $9827 \mathrm{~b}$, c, and d as shown in Figure 4. An order of magnitude calculation of the expected TTV amplitude, based on work by Agol et al. (2005), indicates that the expected amplitude of TTVs is smaller than 3 minutes. Occurring near commensurability of 1:3:5, GJ $9827 \mathrm{c}$ and $\mathrm{b}$ period ratio deviate from $3: 1$ ratio by $+0.5 \%$, whereas the period of GJ $9827 \mathrm{~d}$ and c deviates 5:3 by $+2.0 \%$. Such small positive deviation from the exact resonance has been reported in other Kepler multiple planet systems (Fabrycky et al. 2014). In fact, the period ratio of GJ $9827 \mathrm{c}$ and $\mathrm{d}$ is $1.69994 \pm 0.00003$ $(\sim 1.7)$, where Steffen \& Hwang (2015) reported the presence of a modest peak in their sample of Kepler multiple planet systems. Examples of second-order resonances in our own solar system, as well as in exoplanetary architectures have motivated a dynamical explanation regarding their origin (Mustill \& Wyatt 2011; Xu \& Lai 2017), and a dynamical study of GJ 9827 could be useful in answering questions pertaining to such architecture.

We also phase folded and binned the transit removed data at the period of the first planet to investigate the presence of a phase curve or of a secondary eclipse. None were evident, as the overall noise in the light curve is too dominant to make any statistically significant claim. The GJ 9827 planets may be excellent candidates for searching for such signals in the infrared.

Detected phase curves and secondary eclipse, combined with TTV observations, could help to determine the orbital and planetary parameters with greater precision. The estimated mass of the GJ 9827 super-Earths based on the mass-radius relation proposed by Weiss \& Marcy (2014): $M_{p} / M_{\oplus}=2.69\left(R_{p} / R_{\oplus}\right)^{0.93}$ are $4.5,3.5$, and $5.4 M_{\oplus}$. Based on these mass estimates and orbital parameters, the semi-amplitude of RV signals of the three planets are $3.5,1.9$, and $2.5 \mathrm{~m} \mathrm{~s}^{-1}$. The threshold of $1.5 R_{\oplus}$, as proposed by Weiss \& Marcy (2014), suggests GJ 9827 c to be a rocky, and GJ 9827 d to be a gaseous planet. As for GJ 9827 b, its radius lies close to the boundary itself, and in the light that the exact value of the threshold is not well known (Lopez \& Fortney 2014; Weiss \& Marcy 2014; Rogers 2015), we expect $\mathrm{RV}$ follow-up to shed more light on its density. Details of a concentrated RV campaign will be discussed in a future paper.

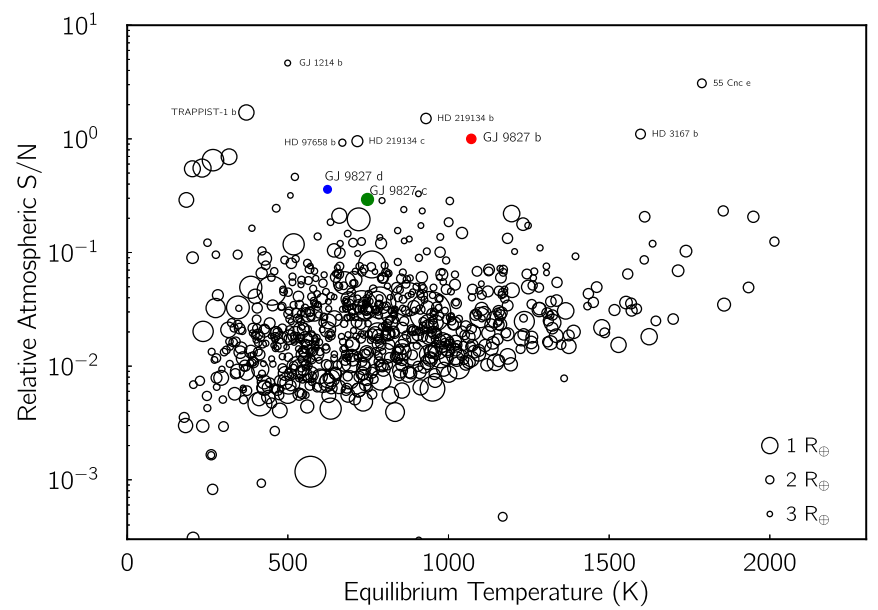

Figure 5. Relative $\mathrm{S} / \mathrm{N}$ of an atmospheric signal for all exoplanet candidates with $R<3 R_{\oplus}$. The GJ 9827 planets are the filled colored symbols with GJ 9827 b used as the $\mathrm{S} / \mathrm{N}$ reference. Using this metric, GJ 9827 b is ranked as the sixth most favorable super-Earth for atmospheric characterization.

\subsection{Prospects for Atmospheric Characterization}

Atmospheric characterization provides an opportunity to not only measure the current conditions in the planetary atmosphere, but also put constraints on formation history and interior structure (Owen et al. 1999), interactions with host star (Cauley et al. 2017), atmospheric and planetary evolution (Öberg et al. 2011), and biological processes (Meadows \& Seager 2010). The planets in the GJ 9827 system offer excellent opportunities to characterize their atmospheres. Figure 5 displays a relative atmospheric detection $\mathrm{S} / \mathrm{N}$ metric (normalized to GJ 9827b) all well characterized with $R_{p}<3 R_{\oplus}$. The sample of small exoplanets, totaling $789,{ }^{21}$ is taken from the NASA Exoplanet Archive. ${ }^{22}$ The atmospheric signal is calculated in a similar way to Gillon et al. (2016) with an effective scale height ( $h_{\text {eff }}=7 H$; Miller-Ricci et al. 2009) using the equilibrium temperature, a Bond albedo of $\alpha=0.3$, and an atmospheric mean molecular weight of $\mu=20$. However, since we calculate the relative signal and assume identical properties for all atmospheres, these values do not affect our results but are included for completeness. The atmospheric signal is dominated by the atmospheric scale height, favoring hot, extended atmospheres, and the host star radius, favoring small, cool stars. The relative $\mathrm{S} / \mathrm{N}$ calculation scales the atmospheric signal with the properties that make it possible to detect and measure this signal,

$$
\begin{gathered}
\frac{\mathrm{S} / \mathrm{N}}{\mathrm{S} / \mathrm{N}_{\mathrm{Ref}}}=\frac{W}{W_{\mathrm{Ref}}} \sqrt{10^{-0.4\left(J-J_{\mathrm{Ref}}\right)}} \sqrt{\frac{P_{\mathrm{Ref}} T_{14}}{P T_{14_{\mathrm{Ref}}}}}, \\
W=\frac{2 R_{p} h_{\mathrm{eff}}}{R_{*}^{2}} .
\end{gathered}
$$

We use the $J$-band flux (e.g., $\mathrm{H}_{2} \mathrm{O}$ measurements with JWST; Beichman et al. 2014), and scale by the duration of the transit and the frequency of transits. Given that sensitive atmospheric observations will likely require many transits to build sufficient signal (e.g., Cowan et al. 2015), we have used a metric that optimizes the $\mathrm{S} / \mathrm{N}$ over a period of time rather than a per-transit metric.

\footnotetext{
21 As of 2017 September 15.

22 https://exoplanetarchive.ipac.caltech.edu
} 
Out of this sample of super-Earth exoplanets, all three planets in the GJ 9827 system are in the top 20 in terms of the $\mathrm{S} / \mathrm{N}$ for atmospheric characterization. This is mainly a consequence of the brightness of this nearby cool, small, star. This highlights the powerful impact nearby stars have on exoplanet characterization given the relative brightness of even small host stars, providing strong atmospheric signals at high $\mathrm{S} / \mathrm{N}$. Using this metric, GJ $9827 \mathrm{~b}$ is ranked the sixth best target for atmospheric characterization, after GJ $1214 \mathrm{~b}$, 55 Cnc e, TRAPPIST-1 b, HD 219134 b, and HD 3167 b. Given that all three of the GJ 9827 planets are near commensurability, there are regular opportunities to observe two, or even all three transits at approximately the same time. For example, see the $K 2$ signal at BJD 2457753, which occurs on average every 150 days (assuming $6 \mathrm{hr}$ of observation). The wait is shorter for simultaneous transits of two planets. Transit overlap occurs for GJ $9827 \mathrm{~b}$ and c over $6 \mathrm{hr}$ of observation on average every 8.7 days; for GJ $9827 \mathrm{c}$ and d around 53 days, and for GJ $9827 \mathrm{~b}$ and d around 15 days.

\section{Conclusion}

Super-Earths are intrinsically interesting objects, as they are universally abundant despite being absent from our solar system. Hosting at least three super-Earths, GJ 9827 lies at a distance of a mere 30 parsecs, the closest planetary system discovered by Kepler or $K 2$. The planets occur on both sides of the rocky gaseous divide; therefore, they are likely to have a different range of densities and provide a test of the precise location of this division. Its three-body second-order resonant system is also intriguing from the viewpoint of planetary architecture and formation. In addition, GJ 9827 is an excellent candidate for follow-up atmospheric characterization with JWST and other facilities. All of these exciting features mean GJ 9827, like other nearby planetary systems around bright stars, will be a great asset for exploring the most fundamental questions of our field.

We are extremely grateful to the NOT staff members for their unique and superb support during the observations. The research leading to these results has received funding from the European Union Seventh Framework Programme (FP7/ 2013-2016) under grant agreement No. 312430 (OPTICON). Based on observations obtained with the Nordic Optical Telescope (NOT), operated on the island of La Palma jointly by Denmark, Finland, Iceland, Norway, and Sweden, in the Spanish Observatorio del Roque de los Muchachos (ORM) of the Instituto de Astrofísica de Canarias (IAC). This paper includes data taken by Kepler. Funding for the Kepler mission is provided by the NASA Science Mission directorate through grant 14-K2G01_2-0071, submitted in response to NNH14ZDA001N Research Opportunities in Space and Earth Science (ROSES-2014). S. Redfield and P. W. Cauley acknowledge the support from the National Science Foundation through Astronomy and Astrophysics Research Grant AST1313268. D. Gandolfi acknowledges the financial support of the Programma Giovani Ricercatori-Rita Levi MontalciniRientro dei Cervelli (2012) awarded by the Italian Ministry of Education, Universities and Research (MIUR). T. Hirano acknowledges support from JSPS KAKENHI Grant Number 16K17660. S. Albrecht and A. B. Justesen acknowledge support by the Danish Council for Independent Research, through a DFF Sapere Aude Starting Grant nr. 4181-00487B.
We also thank the referee for the comments and suggestions that have helped to make this paper better.

During the referee review process, we became aware of a similar discovery in a paper by Rodriguez et al. (2017).

Software: batman (Kreidberg 2015), emcee (ForemanMackey et al. 2013), IRAF (Tody 1986, 1993), k2photometry (Van Eylen \& Albrecht 2015), matplotlib (Hunter 2007), pyaneti (Barragán et al. 2017a), SpecMatch-Emp (Yee et al. 2017).

\section{ORCID iDs}

Prajwal Niraula (iD https://orcid.org/0000-0002-8052-3893 Seth Redfield (i) https://orcid.org/0000-0003-3786-3486 Fei Dai (10) https://orcid.org/0000-0002-8958-0683

Oscar Barragán (i) https://orcid.org/0000-0003-0563-0493 Davide Gandolfi (iD https://orcid.org/0000-0001-8627-9628 P. Wilson Cauley (iD https://orcid.org/0000-0001-9207-0564 Teruyuki Hirano (iD https://orcid.org/0000-0003-3618-7535 Alexis M. S. Smith (iD https://orcid.org/0000-0002-2386-4341 Jorge Prieto-Arranz (i) https://orcid.org/0000-0002-4609-6269 Sascha Grziwa (i) https://orcid.org/0000-0003-3370-4058 Malcolm Fridlund (iD https://orcid.org/0000-0003-2180-9936 Carina M. Persson (iD https://orcid.org/0000-0003-1257-5146 Anders Bo Justesen (i) https://orcid.org/0000-0002-0174-2466 Joshua N. Winn (D) https://orcid.org/0000-0002-4265-047X Simon Albrecht (10 https://orcid.org/0000-0003-1762-8235 William D. Cochran (D) https://orcid.org/0000-00019662-3496

Girish M. Duvvuri (ib https://orcid.org/0000-0002-7119-2543 Michael Endl (i) https://orcid.org/0000-0002-7714-6310 Norio Narita (ib https://orcid.org/0000-0001-8511-2981 Grzegorz Nowak (i) https://orcid.org/0000-0002-7031-7754 Enric Palle (i) https://orcid.org/0000-0003-0987-1593

\section{References}

Agol, E., Steffen, J., Sari, R., \& Clarkson, W. 2005, MNRAS, 359, 567

Alvarado-Gómez, J. D., Hussain, G. A. J., Cohen, O., et al. 2016, A\&A, 594, A95

Armstrong, D. J., Santerne, A., Veras, D., et al. 2015, A\&A, 582, A33

Barragán, O., Gandolfi, D., \& Antoniciello, G. 2017a, pyaneti, Astrophysics Source Code Library, ascl:1707.003

Barragán, O., Gandolfi, D., Smith, A. M. S., et al. 2017b, MNRAS, submitted, arXiv: 1702.00691

Batalha, N. M., Rowe, J. F., Bryson, S. T., et al. 2013, ApJS, 204, 24 Beichman, C., Benneke, B., Knutson, H., et al. 2014, PASP, 126, 1134 Buchhave, L. A., Bakos, G. A., Hartman, J. D., et al. 2010, ApJ, 720, 1118 Carter, J. A., Agol, E., Chaplin, W. J., et al. 2012, Sci, 337, 556

Cauley, P. W., Redfield, S., \& Jensen, A. G. 2017, AJ, 153, 185

Charbonneau, D., Brown, T. M., Noyes, R. W., \& Gilliland, R. L. 2002, ApJ, 568,377

Chiang, E., \& Laughlin, G. 2013, MNRAS, 431, 3444

Cowan, N. B., Greene, T., Angerhausen, D., et al. 2015, PASP, 127, 311

Crossfield, I. J. M., Ciardi, D. R., Petigura, E. A., et al. 2016, ApJS, 226, 7

Crossfield, I. J. M., Petigura, E., Schlieder, J. E., et al. 2015, ApJ, 804, 10

Cutri, R. M., Skrutskie, M. F., van Dyk, S., et al. 2003, yCat, 2246, 0

D’Angelo, G., \& Bodenheimer, P. 2016, ApJ, 828, 33

Deming, D., Seager, S., Winn, J., et al. 2009, PASP, 121, 952

Dressing, C. D., Charbonneau, D., Dumusque, X., et al. 2015, ApJ, 800, 135 Fabrycky, D. C., Lissauer, J. J., Ragozzine, D., et al. 2014, ApJ, 790, 146

Foreman-Mackey, D., Hogg, D. W., Lang, D., \& Goodman, J. 2013, PASP, 125,306

Frandsen, S., \& Lindberg, B. 1999, in Astrophysics with the NOT, FIES: A high resolution Fiber fed Echelle Spectrograph for the NOT, ed. H. Karttunen \& V. Piirola, 71

Fressin, F., Torres, G., Charbonneau, D., et al. 2013, ApJ, 766, 81 Fridlund, M., Gaidos, E., Barragán, O., et al. 2017, A\&A, 604, A16 Gandolfi, D., Barragán, O., Hatzes, A. P., et al. 2017, AJ, 154, 123 
Gandolfi, D., Parviainen, H., Fridlund, M., et al. 2013, A\&A, 557, A74

Gillon, M., Jehin, E., Lederer, S. M., et al. 2016, Natur, 533, 221

Guenther, E. W., Barragan, O., Dai, F., et al. 2017, A\&A, in press, arXiv:1705 04163

Houdebine, E. R., Mullan, D. J., Paletou, F., \& Gebran, M. 2016, ApJ, 822, 97

Howard, A. W., Marcy, G. W., Bryson, S. T., et al. 2012, ApJS, 201, 15

Howell, S. B., Sobeck, C., Haas, M., et al. 2014, PASP, 126, 398

Hunter, J. D. 2007, CSE, 9, 90

Jódar, E., Pérez-Garrido, A., Díaz-Sánchez, A., et al. 2013, MNRAS, 429, 859

Johnson, M. C., Gandolfi, D., Fridlund, M., et al. 2016, AJ, 151, 171

Kipping, D. M. 2010, MNRAS, 408, 1758

Kipping, D. M. 2013, MNRAS, 435, 2152

Knutson, H. A., Charbonneau, D., Allen, L. E., Burrows, A., \& Megeath, S. T. 2008, ApJ, 673, 526

Kovács, G., Zucker, S., \& Mazeh, T. 2002, A\&A, 391, 369

Kreidberg, L. 2015, PASP, 127, 1161

Linsky, J. L., Fontenla, J., \& France, K. 2014, ApJ, 780, 61

Lissauer, J. J., Marcy, G. W., Rowe, J. F., et al. 2012, ApJ, 750, 112

Lissauer, J. J., Ragozzine, D., Fabrycky, D. C., et al. 2011, ApJS, 197, 8

Lopez, E. D., \& Fortney, J. J. 2014, ApJ, 792, 1

Mann, A. W., Feiden, G. A., Gaidos, E., Boyajian, T., \& von Braun, K. 2015, ApJ, 804, 64

McQuillan, A., Aigrain, S., \& Mazeh, T. 2013, MNRAS, 432, 1203

Meadows, V., \& Seager, S. 2010, in Exoplanets, ed. S. Seager (Tucson, AZ: Univ. Arizona Press), 441

Miller-Ricci, E., Seager, S., \& Sasselov, D. 2009, ApJ, 690, 1056

Mustill, A. J., \& Wyatt, M. C. 2011, MNRAS, 413, 554

Öberg, K. I., Murray-Clay, R., \& Bergin, E. A. 2011, ApJL, 743, L16

Owen, T., Mahaffy, P., Niemann, H. B., et al. 1999, Natur, 402, 269

Pecaut, M. J., \& Mamajek, E. E. 2013, ApJS, 208, 9

Ragozzine, D., \& Holman, M. J. 2010, ApJ, in press, arXiv:1006.3727

Redfield, S., Endl, M., Cochran, W. D., \& Koesterke, L. 2008, ApJL, 673, L87
Ricker, G. R., Winn, J. N., Vanderspek, R., et al. 2015, JATIS, 1, 014003

Rodriguez, J. E., Vanderburg, A., Eastman, J. D., et al. 2017, arXiv:1709. 01957

Rogers, L. A. 2015, ApJ, 801, 41

Rowe, J. F., Bryson, S. T., Marcy, G. W., et al. 2014, ApJ, 784, 45

Schlichting, H. E. 2014, ApJL, 795, L15

Sing, D. K. 2010, A\&A, 510, A21

Sing, D. K., Wakeford, H. R., Showman, A. P., et al. 2015, MNRAS, 446, 2428

Sinukoff, E., Howard, A. W., Petigura, E. A., et al. 2016, ApJ, 827, 78

Skrutskie, M. F., Cutri, R. M., Stiening, R., et al. 2006, AJ, 131, 1163

Steffen, J. H., \& Hwang, J. A. 2015, MNRAS, 448, 1956

Stephenson, C. B. 1986, AJ, 92, 139

Telting, J. H., Avila, G., Buchhave, L., et al. 2014, AN, 335, 41

Tody, D. 1986, Proc. SPIE, 627, 733

Tody, D. 1993, in ASP Conf. Ser. 52, Astronomical Data Analysis Software and Systems II, ed. R. J. Hanisch, R. J. V. Brissenden, \& J. Barnes (San Francisco, CA: ASP), 173

Udry, S., Mayor, M., \& Queloz, D. 1999, ASPC, 185, 367

Vanderburg, A., \& Johnson, J. A. 2014, PASP, 126, 948

Vanderburg, A., Johnson, J. A., Rappaport, S., et al. 2015, Natur, 526, 546

Vanderburg, A., Latham, D. W., Buchhave, L. A., et al. 2016, ApJS, 222, 14

Van Eylen, V., \& Albrecht, S. 2015, ApJ, 808, 126

Van Eylen, V., Albrecht, S., Gandolfi, D., et al. 2016, AJ, 152, 143

van Leeuwen, F. 2007, A\&A, 474, 653

Weiss, L. M., \& Marcy, G. W. 2014, ApJL, 783, L6

Wood, B. E., Müller, H.-R., Zank, G. P., Linsky, J. L., \& Redfield, S. 2005 , ApJ, 628, 143

Wright, E. L., Eisenhardt, P. R. M., Mainzer, A. K., et al. 2010, AJ, 140, 1868

Xu, W., \& Lai, D. 2017, MNRAS, 468, 3223

Yee, S. W., Petigura, E. A., \& von Braun, K. 2017, ApJ, 836, 77

Zacharias, N., Finch, C. T., Girard, T. M., et al. 2013, AJ, 145, 44 